\title{
Hypoxia in utero increases the risk of pulmonary hypertension in rat offspring and is associated with vasopressin type- 2 receptor upregulation
}

\author{
HAO DING ${ }^{1,2}$, YUCHUAN LUO ${ }^{2}, \mathrm{KE} \mathrm{HU}^{2}$, HUAYING HUANG ${ }^{1}$, PEI LIU ${ }^{2}$, \\ MENGQING XIONG ${ }^{2}$, LI ZHU ${ }^{1}$, JING YI $^{1}$ and YING XU ${ }^{1}$ \\ ${ }^{1}$ Division of Respiratory Disease, Affiliated People's Hospital of Jiangsu University, Zhenjiang, Jiangsu 212002; \\ ${ }^{2}$ Division of Respiratory Disease, Renmin Hospital of Wuhan University, Wuhan, Hubei 430060, P.R. China
}

Received May 18, 2018; Accepted July 28, 2020

DOI: $10.3892 / \mathrm{mmr} .2020 .11533$

\begin{abstract}
Pulmonary hypertension (PH) in newborns and adults is a disease that can lead to right heart failure and result in a shorter lifespan. $\mathrm{PH}$ was induced by maintaining pregnant rats in a hypoxic chamber for $4 \mathrm{~h}$ twice a day, from days 7-21 of pregnancy. Hypoxia was confirmed by a decrease in the partial pressure of oxygen $\left(\mathrm{PaO}_{2}\right)$ and the oxygen saturation $\left(\mathrm{SaO}_{2}\right)$ of arterial blood in the aorta. The body weight of newborns from hypoxic rats was $\sim 20 \%$ decreased compared with the control newborns of normoxic rats. The vascular wall thickness/vascular diameter values of hypoxia treated pubs were increased compared with that of control newborns 7 days after birth; however, it decreased to similar levels than in the control group after 3 months, and then further decreased to significantly lower levels than in the control group at 6 months after birth. At birth, the lung tissues of newborns from hypoxic rats exhibited an increase in the levels of mRNA and proteins associated with $\mathrm{PH}$ such as HIF- $1 \alpha$, HIF- $2 \alpha$, V2R, TGF- $\beta$, TNF- $\alpha$, Ang- 2 and $\alpha$-SMA. At 3 and 6 months after birth, the levels of both V2R mRNA and protein in offspring from hypoxic rats were at least 2-fold higher, whereas the expression of all other factors decreased compared with the control offspring. By contrast, HIF-2 $\alpha$ and Ang-2 expression levels were significantly increased in the 6-month-old control offspring from normoxic rats. V2R overexpression in pups induced by hypoxia in maternal rats was sustained until their adulthood. V2R may be a marker for detecting $\mathrm{PH}$.
\end{abstract}

Correspondence to: Professor $\mathrm{Ke} \mathrm{Hu}$, Division of Respiratory Disease, Renmin Hospital of Wuhan University, 99 Zhangzhidong Road, Wuhan, Hubei 430060, P.R. China

E-mail: hukewuhan@163.com

Key words: pulmonary hypertension, vasopressin type-2 receptor, offspring, hypoxia, pregnant rats

\section{Introduction}

During pregnancy, the lungs of the fetus do not exchange oxygen $\left(\mathrm{O}_{2}\right)$ and carbon dioxide $\left(\mathrm{CO}_{2}\right)$, and therefore require a minimal blood supply. When the baby is born, it breathes by itself. The lungs dilate, decreasing the pulmonary vascular resistance and left atrium pressure considerably. However, certain newborns are unable to decrease their pulmonary pressure due to prenatal or postnatal factors (1).

The hypoxic level of blood that perfuses the fetal lungs is perhaps the major determinant of the degree of vasoconstriction of the pulmonary arterioles. Muscle fibers in pulmonary arterioles are highly sensitive to oxygen levels and changes in $\mathrm{pH}$, contract under acidotic and hypoxic conditions, and vasodilate when there are changes to the partial pressure of oxygen $\left(\mathrm{PaO}_{2}\right)$ and $\mathrm{pH}$ levels (2). Pulmonary vascular disease may affect the pulmonary capillaries and/or arterioles responsible for pulmonary arterial hypertension, or affect the left ventricle, leading to pulmonary venous hypertension. Pulmonary arterial hypertension is characterized by dysfunction of endothelial cells and proliferation of intimal and smooth muscle cells. Pulmonary vascular resistance and pressure has a deleterious effect on the right ventricle (RV), with a resulting decrease in cardiac output and right heart failure (HF), accompanied by the patient having a decreased ability to exercise and in the most serious cases, patient mortality (3-6).

In addition, it is likely that a number of epigenetic changes are involved in the regulation of the perinatal pulmonary circulation (7-9). Factors involved in pulmonary hypertension $(\mathrm{PH})$ include hypoxia inducible factor $1 \alpha(\mathrm{HIF}-1 \alpha)(10)$, transforming growth factor $\beta$ (TGF- $\beta$ ) (11), tumor necrosis factor $\alpha$ (TNF- $\alpha)(12-14)$, soluble endoglin and placental growth factor (PlGF) (15), angiopoetin-2 (Ang-2) (16) and $\alpha$ smooth muscle actin ( $\alpha$-SMA) protein (17).

Furthermore, pulmonary arteriole vasodilatation during birth is generally inversely correlated with the muscular tunic thickness at the end of the gestational period. However, the muscular tunic is reabsorbed postnatally, a process that occurs during the first 14 days of life. Occasionally, this reabsorption may take a considerably longer period of time. This timeframe may have a negative effect on the necessary adjustments to the 
postnatal circulation, as the thickness of the muscular tunic limits the lumen diameter, rendering pulmonary arterioles more reactive to stimuli that trigger vasoconstriction $(18,19)$, which may negatively affect the therapeutic outcome.

Pulmonary arterial hypertension, however, is a condition that may ultimately trigger right HF. Prostaglandin $\mathrm{I}_{2}$-targeted drugs are currently used for vascular endothelial proliferation, expansion of pulmonary vessels and to inhibit pulmonary vascular remodeling (20). Endothelin receptor antagonists serve a role in the expansion of pulmonary vessels, anti-proliferation and endothelial function improvements. Phosphodiesterase type 5 (PDE5) inhibitors prevent the general expression of PDE5 in PH by increasing the levels of cGMP in vascular smooth muscle cells. cGMP triggers dilation of vascular smooth muscle, leading to an expansion of the pulmonary artery lumen and a decrease in pulmonary vascular resistance, thereby decreasing pulmonary arterial pressure (PAP). A previous study has demonstrated that arginine vasopressin (AVP) is released from the posterior pituitary gland when there is a decrease in the circulating blood volume $(21,22)$. AVP may induce vasopressin type-2 receptor (V2R) expression, while previous data has suggested that the long-term administration of V2R antagonists has beneficial actions on cardiac hemodynamics, and improves myocardial fibrosis and left ventricular (LV) remodeling together with a diuretic effect in left HF $(18,19)$. However, the prognostic effect of PH and RV dysfunction in LV function is of critical importance in determining precisely the cause and severity of $\mathrm{PH}$ prior to making decisions concerning treatment. Therefore, a comprehensive diagnostic strategy is required, but targeted HF treatments (medical/interventional) remain limited (3). Therefore, the goal of treatment is to delay or prevent the progression of $\mathrm{PH}$. Although its incidence is not high at present, it is an easily misdiagnosed disease. The incidence of $\mathrm{PH}$ in the newborn is 0.43-6.6 per 1,000 live offspring and most commonly occurs in preterm newborns (23-25). In adults, the average age of onset is 36 years, with $75 \%$ of patients within the 20-40 years age category. The onset of $\mathrm{PH}$ in adulthood may be associated with the hypoxic intrauterine environment $(26,27)$. A possibility is that the expression pattern of molecular markers associated with $\mathrm{PH}$ and hypoxia, and associated factors induced by hypoxia in utero, remain unchanged in adulthood.

The objectives of the present study were to identify the physiological roles of factors associated with pulmonary artery hypertension including V2R, HIF- $1 \alpha$, HIF- $2 \alpha$, TGF- $\beta$, PIGF and other angiogenesis factors. To explore the mechanism of $\mathrm{PH}$ induction for diagnostic and therapeutic possibilities, the expression of these factors was measured in newborns and in later months (corresponding to adulthood in the human), utilizing a rat model in which maternal rats lived in a hypoxic environment during their pregnancy.

\section{Materials and methods}

Animals. All experiments on rats were conducted according to the guidelines for the Humane Treatment of Laboratory Animals (Ministry of Science and Technology of the People's Republic of China, Policy. No. 2006 398) (28) and additional approval for the present study was waived by the Ethics Committee of the Affiliated People's Hospital of Jiangsu
University (Zhenjiang, China). A total of 20 3-month old female Sprague Dawley rats, kindly provided by the Shanghai Experimental Animal Center (Shanghai, China), and were housed under standard laboratory conditions $\left(22 \pm 2^{\circ} \mathrm{C}\right.$, $60 \%$ relative humidity, $12 / 12 \mathrm{~h}$ light/dark cycle and access to food and water ad libitum). The rats were mated and vaginal smears taken each morning were examined. Day zero of a pregnancy duration of 22 days was denoted when the presence of sperm was observed. During each rat's pregnancy and period of lactation, all experimental animals had ad libitum access to standard laboratory water and food. On day 7 of pregnancy, the animals were assigned randomly into control (normoxic) and experimental (hypoxic) groups (Fig. 1).

Hypoxic treatment of maternal rats. From days 7 to 21 of pregnancy, the rats were allocated to the control or hypoxic groups. The rats in the hypoxic group were placed in a hypoxic chamber in which the oxygen supply was reduced to $10 \pm 1 \%$ by continuous infusion of nitrogen gas and compressed air mixture, while expired $\mathrm{CO}_{2}$ was eliminated by circulating the atmosphere through soda lime. The water contained in the expired gas was trapped in a chilled glass tank. A portable oxygen analyzer (S-450; IST-AIM) was calibrated daily and used to monitor the oxygen concentration of the chamber as described previously (29).

Blood gases and the $\mathrm{pH}$ of arterial blood were measured in aliquots of blood withdrawn after $1 \mathrm{~h}$ exposure to hypoxia from a cannula positioned in the left femoral artery. Measurements were performed on 3 randomly selected rats using a Rapidlab 850 analyzer (Bayer AG) that automatically measured blood gas levels. After $4 \mathrm{~h}$ of hypoxia, the pregnant rats were released from the hypoxia chamber to normal room air. A total of 10 rats (controls) were placed in an identical environment under normoxic conditions. The rats allocated to the hypoxia group were exposed to a hypoxic environment twice a day for $4 \mathrm{~h}$ in the morning and $4 \mathrm{~h}$ in the afternoon, for a total of 2 weeks, with a break for 1 day after 6 days (Fig. 1).

Measurement of pulmonary artery pressure and the RV hypertrophy index. Following anoxic treatment for 1 and 3 weeks, maternal rats were anesthetized with an intraperitoneal injection of $50 \mathrm{mg} / \mathrm{kg}$ pentobarbital sodium $(30,31)$. A catheter was introduced into the RV and the pulmonary artery via the right external jugular vein. A pressure sensor connected to the multi-guide physiological recorder was used to make measurements of the pulmonary artery and to compute the mean PAP. Then, the animals were sacrificed by exsanguination. In the neonates ( 7 days old), $0.5-1 \mathrm{ml}$ blood was removed. In the 3- and 6-month-old rats, $8-10 \mathrm{ml}$ blood was removed. The animals were then decapitated, and the heart and lung tissues were separated and the blood in the heart was rinsed away with PBS buffer. The atrium, aorta and pulmonary artery were cut off along the atrioventricular canal. The RV free wall was separated along the anterior interventricular sulcus.

Offspring selection and grouping. From each litter, 1 pup was randomly selected and the heart, lungs, liver, kidney and brain tissues were dissected and weighed between 3 and $12 \mathrm{~h}$ after birth. The size of the litter was decreased to 8 experimental animals to ensure that the offspring had equal access 


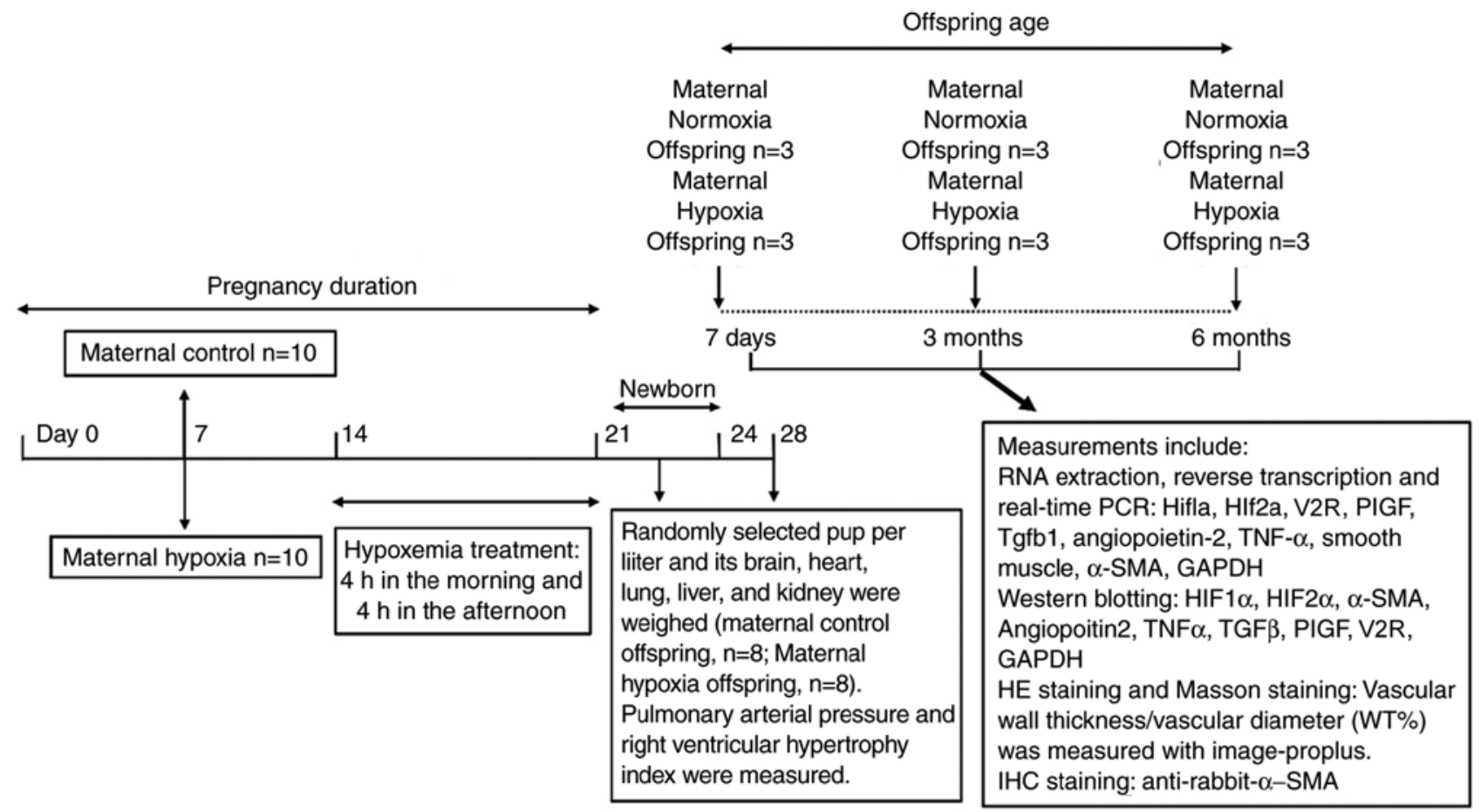

Figure 1. Schematic diagram of rat maternal hypoxia treatment and measurement methods in different offspring age groups. HIF-1 $\alpha$, hypoxia-inducible factor-1 $\alpha$; HIF-2 $\alpha$, hypoxia-inducible factor-2 $\alpha$; V1R, vasopressin type-1 receptor; V2R, vasopressin type-2 receptor; PLGF, placental growth factor; TGF- $\beta$, Transforming growth factor- $\beta 1$; Ang- 2 , angiopoietin- 2 ; TNF- $\alpha$, tumor necrosis factor- $\alpha$; $\alpha$-SMA, $\alpha$-smooth muscle aorta.

to nutrients. The organs from 1 randomly selected pup in each litter were weighed after 28 days. The offspring of 10 control and 10 hypoxia litters were randomly assigned into 6 groups of 3 rats: Maternal; normoxia; and hypoxia rat groups, at 7 days, 3 months and 6 months after birth. In total, 20 rats, including 2 extra rats, were used in the experiments. The tissues of 10 rats in each group, which were resected at the indicated time points, were immediately cut into small pieces for freezing. Different sections were used for RNA extraction, protein extraction and hematoxylin \& eosin (H\&E) and Masson's staining following slicing. For each experiment, 3 samples were used from different rats and each sample was measured 3 times. The offspring rats (female and male) were randomly placed in each group with at least 1 female rat and 1 male rat in one group without any differences in appearance (color or size).

Extraction of RNA, reverse transcription and quantitative $(q)$ $P C R$ to identify target factors. The frozen lung tissue of rats exposed to hypoxic conditions ( 7 days, 3 months and 6 months after birth) was kept in $1 \mathrm{ml} \mathrm{TRIzol}{ }^{\circledR}$ in a clean homogenate tube. The tissue was homogenized for $20 \mathrm{sec}$ and centrifuged at $13,400 \times \mathrm{g}$ for $10 \mathrm{~min}$ at room temperature. Subsequently, the isolated supernatant was transferred into fresh $1.5 \mathrm{ml}$ centrifuge tubes and $200 \mu \mathrm{l}$ chloroform and $600 \mu \mathrm{l}$ isopropyl alcohol were added. Following centrifugation $(13,400 \mathrm{x} \mathrm{g}$, $\left.10 \mathrm{~min}, 4^{\circ} \mathrm{C}\right)$, the precipitate was washed in ethanol $(75 \%)$ and the extracted RNA was subsequently dissolved in diethylpyrocarbonate-treated water.

A SuperScript II reverse transcriptase kit (Invitrogen; Thermo Fisher Scientific, Inc.) was used to measure total RNA. qPCR was performed using SYBR Green Supermix and
Premix Ex Taq ${ }^{\mathrm{TM}}$ kits (Takara Biotechnology Co., Ltd.) and an iCycler system (Bio-Rad Laboratories, Inc.). A quantity of $2 \mu 1$ was then mixed with $12.5 \mu \mathrm{l}$ SYBR Green reaction master mix, $0.5 \mu \mathrm{l}$ forward and $0.5 \mu \mathrm{l}$ reverse primers. The thermocycler conditions were as follows: Initial heating at $95^{\circ} \mathrm{C}$ for $10 \mathrm{~min}$; then 40 cycles of denaturation: $95^{\circ} \mathrm{C}$ for $15 \mathrm{sec}$; annealing and elongation: $60^{\circ} \mathrm{C}$ for $45 \mathrm{sec}$ without final extension. Melt curve analysis: $95^{\circ} \mathrm{C}$ for $15 \mathrm{Sec} ; 60^{\circ} \mathrm{C}$ for $1 \mathrm{~min} ; 95^{\circ} \mathrm{C}$ for $15 \mathrm{Sec}$; $60^{\circ} \mathrm{C}$ for $15 \mathrm{Sec}$. The sequences of forward and reverse primers used are provided in Table I.

The levels of gene expression were normalized to GAPDH, which was used as the housekeeping gene. Each reaction was performed in triplicate and qPCR was carried out with the aid of a sequence detection system (ABI 7300; Applied Biosystems; Thermo Fisher Scientific, Inc.). The data are presented as means \pm standard deviation (SD) from at least 3 separate measurements. Expression levels, again normalized to GAPDH expression, were determined using the $2^{-\Delta \Delta \mathrm{Cq}}$ method (32).

Western blotting. The frozen lung tissue was cut into small fragments. Then 150-250 $\mu \mathrm{l}$ lysis buffer (cat. no. R0010, Beijing Solarbio Science \& Technology Co., Ltd.) containing protease and phosphatase inhibitors was added to a $20 \mathrm{mg}$ tissue sample, which was subsequently thoroughly homogenized. The homogenate was spun at $12,000 \mathrm{x} \mathrm{g}$ for $15 \mathrm{~min}$ at $4^{\circ} \mathrm{C}$, and the separated supernatant protein concentration measured using the BCA assay (Thermo Fisher Scientific, Inc.). Equal quantities of protein $(10 \mu \mathrm{g})$ were separated in $8 \%$ denaturing polyacrylamide gel and then electro transferred onto a nitrocellulose membrane (cat. no. 1620115; Bio-Rad Laboratories, Inc.). Following blocking at room 
Table I. Primer sequences of the indicated target genes.

Genes

HIF-1 $\alpha$ (NM_001313919.1)

HIF-2 $\alpha$ (NM_010137.3)

V1R (NM_016847.2)

V2R (NM_001276298.1)

PIGF (NM_008838.1)

TGF- $\beta$ (NM_011577.2)

Ang-2 (NM_007426.4)

TNF- $\alpha$ (NM_001278601.1)

Q-SMA (NM_007392.3)

GAPDH (NM_001289726.1)

Primer sequences

F: 5'TCTCGGCGAAGCAAAGAGTC3'
R: 5'AGCCATCTAGGGCTTTCAGATAA3'
F: 5'GAGGAAGGAGAAATCCCGTGA3'
R: 5'TATGTGTCCGAAGGAAGCTGA3'
F: 5'-TCGTGGTGGCCGTGCTGGGT-3'
R: 5'-ACCGCCAGGTCTGCCAGGCT-3'
F: 5'ATCCTGGTGTCTACCACGTCT3'
R: 5'GTTCAGCCCGGACTAACAGC3'
F: 5'TCCTTCTTCGTGGACAACTTCT3'
R: 5'GTACGACAAGAGATTGACAGTGG3'
F: 5'CCACCTGCAAGACCATCGAC3'
R: 5'CTGGCGAGCCTTAGTTTGGAC3'
F: 5'CAGCCACGGTCAACAACTC3'
R: 5'CTTCTTTACGGATAGCAACCGAG3'
F: 5'GTGCTCAGAGCTTTCAAC3'
R: 5'ACTCTCCCTTTGCAGAAC3'
F: 5'CCCAGACATCAGGGAGTAATGG3'
R: 5'TCTATCGGATACTTCAGCGTCA3'
F: 5'CTGCCCAGAACATCATCC3'
R: 5'CTCAGATGCCTGCTTCAC3'

F, forward; R, reverse; HIF-1 $\alpha$, hypoxia-inducible factor- $1 \alpha$; HIF-2 $\alpha$, hypoxia-inducible factor-2 $\alpha$; V1R, vasopressin type-1 receptor; V2R, vasopressin type-2 receptor; PlGF, placental growth factor; TGF- $\beta$, Transforming growth factor- $\beta$ 1; Ang- 2 , angiopoietin- 2 ; TNF- $\alpha$, tumor necrosis factor- $\alpha$; $\alpha$-SMA, $\alpha$-smooth muscle aorta.

overnight at $4^{\circ} \mathrm{C}$ in TBS-T (containing fat-free milk 5\%), membranes were incubated with the following primary antibodies: Mouse anti-HIF-1 $\alpha$ (1:500, cat. no. Ab113642; Abcam); rabbit anti-HIF-2 $\alpha$ (1:1,000, cat. no. Ab109616; Abcam); rabbit anti- $\alpha$-SMA (1:300, cat. no. Ab5694; Abcam); rabbit anti-Ang-2 (1:1,000, cat. no. Ab180820; Abcam); rabbit anti-TNF- $\alpha$ (1:1,000, cat. no. Ab6671; Abcam); rabbit anti-TGF- $\beta$ (1:400, cat. no. Ab92486, Abcam); mouse anti-PIGF (1:800, cat. no. RM0010-8F09; Novus Biologicals, LLC); rabbit anti-V2R (1:500, cat. no. ab176488; Abcam); and rabbit anti-GAPDH (1:2,000, cat. no. 5174; Cell Signaling Technology, Inc.), overnight at $4^{\circ} \mathrm{C}$. Then the membranes were washed by TBS-T for 5 min three times at room temperature. They were then incubated with horseradish peroxidase (HRP)-conjugated goat anti-rabbit IgG (1:1,000, cat. no. A0208) and goat anti-mouse $\operatorname{IgG}(1: 1,000$, cat. no. A0216) (both from Beyotime Institute of Biotechnology) secondary antibodies for $1 \mathrm{~h}$ at $37^{\circ} \mathrm{C}$, and bands were measured using an ECL detection system (GE Healthcare Life Sciences). Densitometric analysis was performed using Image-Pro Plus 6.0 software (Media Cybernetics, Inc.) to quantify the band expression levels, which were expressed as the band density to total GAPDH ratio.

$H \& E$ staining. The frozen lung tissues, which had been stored at $-80^{\circ} \mathrm{C}$, were placed at $-20^{\circ} \mathrm{C}$ for $30 \mathrm{~min}$ and then sliced into $5-7-\mu \mathrm{m}$-thick sections using a freezing microtome
(Leica Microsystems $\mathrm{GmbH}$ ). The sections were carefully attached to the slides and immobilized in acetone at $4{ }^{\circ} \mathrm{C}$ for $15 \mathrm{~min}$. The sections were then washed with distilled water and placed for $5 \mathrm{~min}$ in hematoxylin solution (cat. no. 714094, BaSO Biotech Co., Ltd.) at room temperature for staining, and then washed with ammonia water. After a few seconds, the sections were washed with running water for $15 \mathrm{~min}$ and dehydrated in 70 and $90 \%$ alcohol for 10 min each at room temperature. Sections were then stained with ethanol eosin staining solution (cat. no. BA4099, BaSO Biotech Co., Ltd.) at room temperature for 1-2 min. The stained sections were dehydrated in $99.7 \%$ ethanol and the slides were vitrified in dimethyl benzene twice for $3 \mathrm{~min}$. The samples were placed in a $65^{\circ} \mathrm{C}$ oven for $15 \mathrm{~min}$ after mounting the slides in neutral balsam and viewed using a CX41 light microscope at magnification x400 (Olympus Corporation).

Masson's trichrome staining. Each section was attached to a slide coated with poly-l-lysine and then immobilized for $20 \mathrm{~min}$ in $4 \%$ acetone solution and washed 3 times with distilled water for $3 \mathrm{~min}$. Then the Masson's Trichrome Staining Kit (cat. no. DC0032; Leagene Biotech Co., Ltd.) was used according to the manufacturer's protocol. The section was subsequently stained with freshly prepared Weigert iron hematoxylin solution (hematoxylin solution/ferric chloride solution=1:1) at room temperature for 5-10 $\mathrm{min}$ and then differentiated with acidic ethanol differentiation solution. 
Following washing, the section was treated with Masson blue solution and then stained with aniline blue counterstain solution at room temperature for 1-2 min after washing the section with weak acid working fluid and phosphate molybdenum acid. Finally, the sections were mounted in neutral balsam after vitrifying them in dimethylbenzene 3 times and viewed with a CX41 light microscope at magnification $\mathrm{x} 400$ (Olympus Corporation).

Percentage of pulmonary vascular wall thickness to vascular diameter. H\&E-stained sections from mouse lung tissues were observed using a light microscope at magnification, $\mathrm{x} 400$. A total of 5 small and medium-sized arteries with relatively round cross sections were selected. The vascular wall thickness/vascular diameter (WT\%) was determined using Image-Pro Plus 6.0 software (Media Cybernetics, Inc) and the average values were calculated.

Immunohistochemistry staining. The sections were attached to slides coated with poly-l-lysine. Then the sections were placed in $3 \% \mathrm{H}_{2} \mathrm{O}_{2}$ and incubated in a wet box for $10 \mathrm{~min}$ to block endogenous peroxidase activity. Sections were then washed 3 times in $0.02 \mathrm{M}$ PBS for $3 \mathrm{~min}$ and primary antibodies (anti-rabbit- $\alpha$-SMA; 1:200, cat. no. Ab5694; Abcam) were added. Sections were incubated in a wet box and stored at room temperature for $1 \mathrm{~h}$ or overnight at $4^{\circ} \mathrm{C}$. Then they were washed 3 times in $0.02 \mathrm{M}$ PBS for $3 \mathrm{~min}$ and HRP-conjugated secondary antibodies (1:50, cat. no. D-3004, Shanghai Long Island Biotec Co., Ltd.) were applied. They were incubated for 20-30 min at room temperature, washed with PBS, and then stained using a DAB kit (Shanghai Long Island Biotec. Co., Ltd; Mindray Medical), and counterstained with hematoxylin (BaSO Biotech Co., Ltd) for $3 \mathrm{~min}$. They were differentiated in $1 \%$ hydrochloric acid alcohol, vitrified in $100 \%$ dimethyl benzene, mounted in neutral balsam and observed using a CX41 light microscope (Olympus Corporation).

Statistical analysis. SPSS for Windows v.16.0 (SPSS Inc.) was used for statistical analysis of the data. Data are presented as the mean \pm SD. A Student's t-test was used to analyze the arterial blood gas levels $\left[\mathrm{PH}\right.$, arterial $\mathrm{CO}_{2}$ partial pressure $\left(\mathrm{PaCO}_{2}\right), \mathrm{PaO}_{2}$ and $\mathrm{SaO}_{2}$ ], body weight and artery pressure. Comparisons between the relative levels of mRNA and protein of target genes, the ratio of vascular wall thickness and vascular diameter and positive counts of $\alpha$-SMA staining between the control and hypoxia groups in different treatment days were measured by two-way ANOVA and Bonferroni post hoc test. All tests were two-sided and $\mathrm{P}<0.05$ was considered to indicate a statistically significant difference.

\section{Results}

Characteristics of offspring. Firstly, the levels of $\mathrm{PaCO}_{2}$, $\mathrm{PaO}_{2}, \mathrm{SaO}_{2}$ and $\mathrm{pH}$ in the blood of maternal pregnant rats were determined after $1 \mathrm{~h}$ of hypoxia treatment (Table II). The results showed that hypoxia treatment significantly affected the concentrations of $\mathrm{PaO}_{2}$ and $\mathrm{SaO}_{2}$ (Table II).

It was confirmed that 3 weeks of intermittent hypoxia promoted the development of typical PH and RV hypertrophy in the rats. These results indicated that the hypoxia $\mathrm{PH}$ rat
Table II. Arterial blood gas levels in maternal pregnant control rats and hypoxic pregnant rats after hypoxia treatment for $1 \mathrm{~h}$.

\begin{tabular}{lccc}
\hline Parameters & Control $(\mathrm{n}=3)$ & Hypoxia $(\mathrm{n}=3)$ & P-value \\
\hline $\mathrm{PH}$ & $7.42 \pm 0.15$ & $7.33 \pm 0.18$ & 0.369 \\
$\mathrm{PaCO}_{2}, \mathrm{kPa}$ & $5.67 \pm 1.43$ & $7.38 \pm 2.12$ & 0.133 \\
$\mathrm{PaO}_{2}, \mathrm{kPa}$ & $10.98 \pm 1.22$ & $6.58 \pm 2.13$ & 0.001 \\
$\mathrm{SaO}_{2}, \%$ & $94.50 \pm 2.30$ & $70.50 \pm 14.20$ & 0.002 \\
\hline
\end{tabular}

All data are expressed as means \pm standard deviation for control $(n=3)$ and hypoxic $(\mathrm{n}=3)$ rats. $\mathrm{PaO}_{2}$, arterial $\mathrm{O}_{2}$ partial pressure; $\mathrm{PaCO}_{2}$, arterial $\mathrm{CO}_{2}$ partial pressure.

Table III. Effect of maternal hypoxia on weights and artery pressure of newborns.

\begin{tabular}{lccc}
\hline Parameters & Control & Hypoxia & P-value \\
\hline Body weight, g & $5.86 \pm 0.31$ & $4.87 \pm 0.51$ & $<0.01$ \\
Average Rvp, kPa & $2.56 \pm 0.28$ & $5.05 \pm 0.65$ & $<0.05$ \\
Average PAP, $\mathrm{kPa}$ & $2.86 \pm 0.57$ & $5.38 \pm 0.65$ & $<0.05$ \\
Lung weight to body & $2.21 \pm 0.63$ & $2.03 \pm 0.59$ & $>0.05$ \\
weight, \% & & & \\
\hline
\end{tabular}

All data are expressed as means \pm standard deviation for control $(n=3)$ and hypoxic $(n=3)$ rats. Rvp, right ventricular pressure; PAP, pulmonary artery pressure.

model was successfully established (data not shown). In the newborns from the hypoxic rat group, significant differences in body weights of the newborns on day 21 ( $20 \%$ lighter) compared with the newborns from control rats were observed (Table III). Significant differences in RV pressure (Rvp) and PAP were also observed; these values in the offspring from hypoxic rats were decreased by approximately one-half of those in the offspring from the control group. However, the ratios of lung weight to body weight between the groups were not statistically different.

Changes in epigenetic factors involved in the control of the pulmonary circulation of the neonate. The mRNA expression levels of HIF- $1 \alpha$, HIF- $2 \alpha$, TGF- $\beta$, TNF- $\alpha$, PIGF, Ang- $2, \alpha-$ SMA, $\mathrm{V} 1 \mathrm{R}$ and $\mathrm{V} 2 \mathrm{R}$ in the lung tissues of newborns, and 3-monthand 6-month-old rats were measured using RT-qPCR (Fig. 2). The expression levels of all of these mRNAs were increased in rats that had been exposed to a hypoxic environment. This may be due to the activation of HIF induced by long-term hypoxia treatment and the activation of angiogenic factors regulated by HIF. However, the expression of these factors were mostly normalized 3 months after birth. Only the levels of V2R and Ang-2 exhibited significant differences between the two groups at 3 months after birth, with increased expression levels in the hypoxia groups. The transcription levels of the other mRNAs were all significant increased between in the hypoxia compared to the control group at 7 days after birth. As the increase in V2R mRNA transcription levels was sustained 

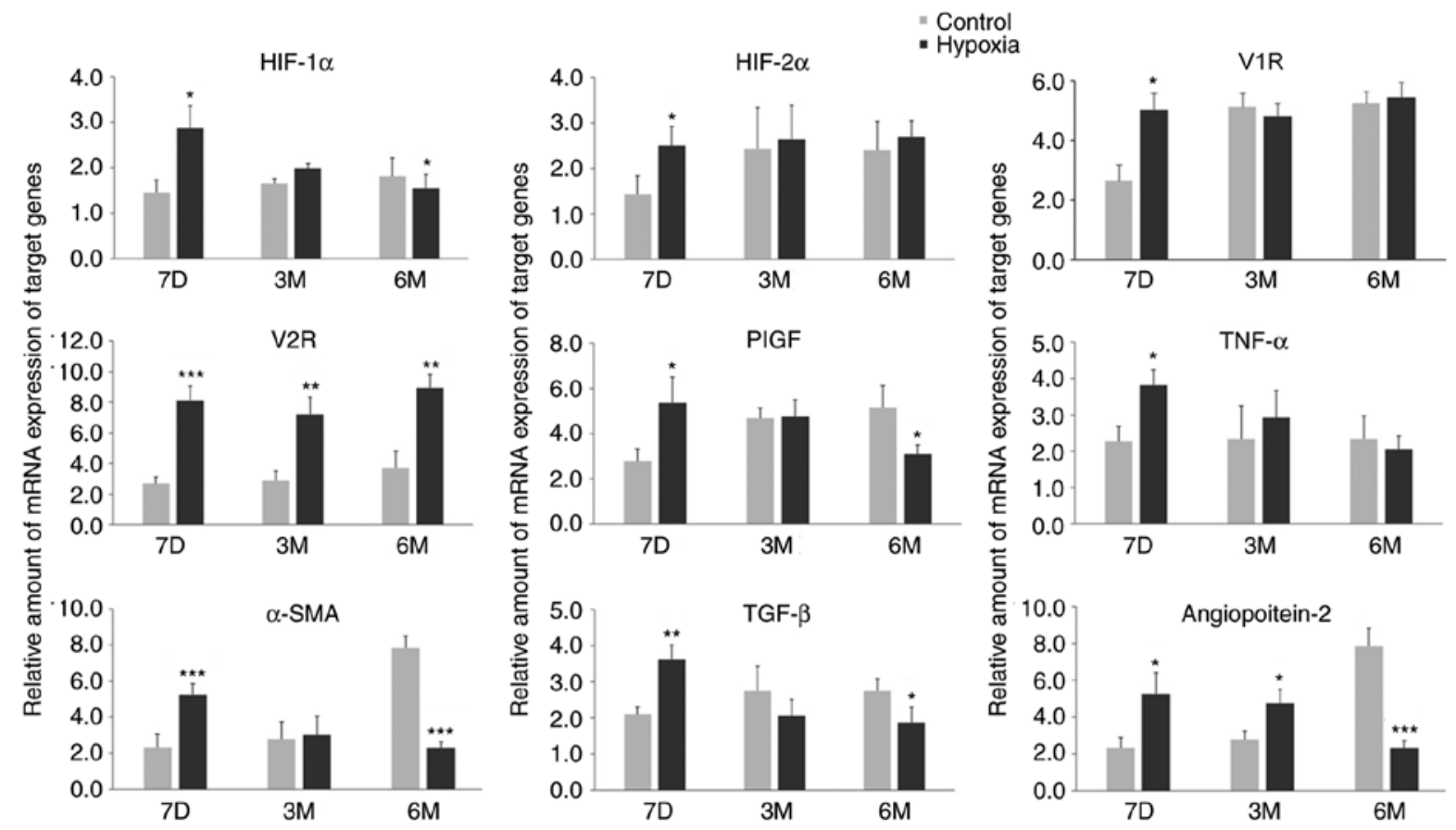

Figure 2. Maternal rats were exposed to continuous hypoxia from days 7-21 during pregnancy and the relative levels of mRNA of target genes in lung tissues of pups at 7D, 3M and 6M after birth were measured. $\mathrm{n}=3$. Samples were each measured 3 times. Data are presented as mean \pm standard deviation. A two-way ANOVA and Bonferroni post hoc test were used to analyze the data between the control and hypoxia groups. ${ }^{*} \mathrm{P}<0.05,{ }^{* *} \mathrm{P}<0.01$ and ${ }^{* * * *} \mathrm{P}<0.001$ vs. control. HIF- $1 \alpha$, hypoxia-inducible factor-1 $\alpha$; HIF-2 $\alpha$, hypoxia-inducible factor- $2 \alpha$; V1R, vasopressin type-1 receptor; V2R, vasopressin type-2 receptor; PIGF, placental growth factor; TGF- $\beta$, Transforming growth factor- $\beta$ 1; TNF- $\alpha$, tumor necrosis factor- $\alpha$; $\alpha$-SMA, $\alpha$-smooth muscle aorta; 7D, 7 days; $3 \mathrm{M}, 3$ months; $6 \mathrm{M}, 6$ months.
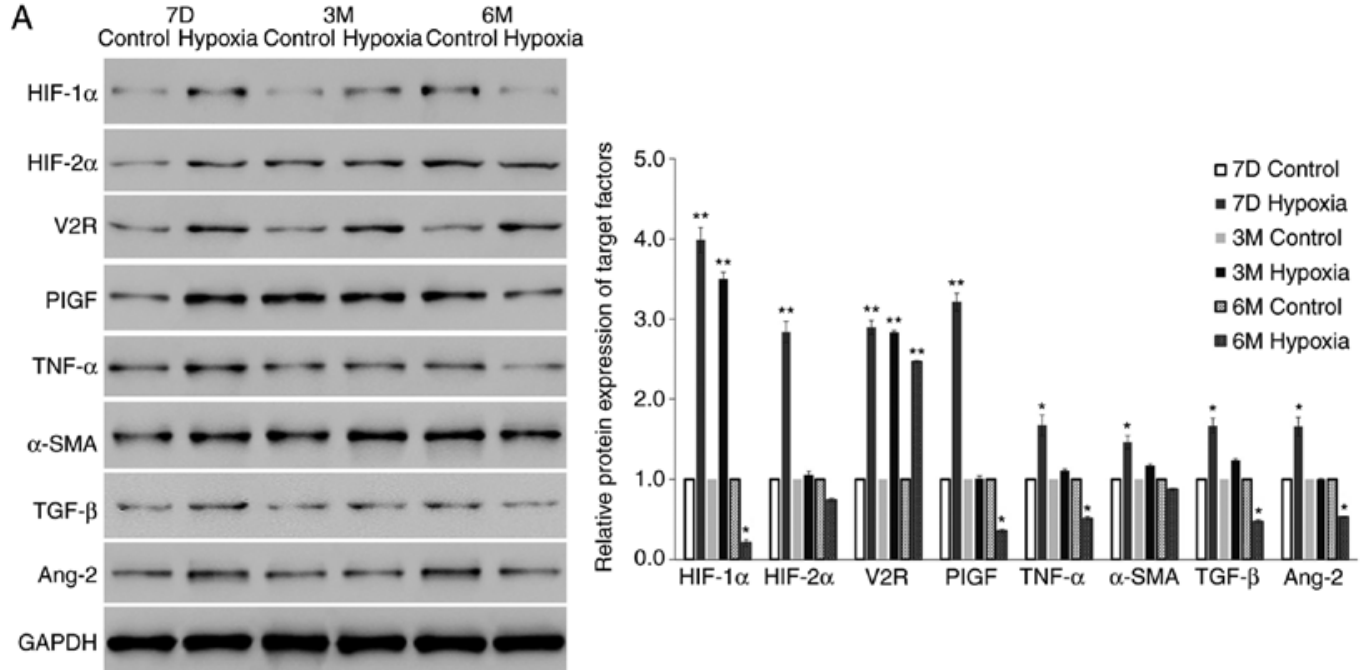

B
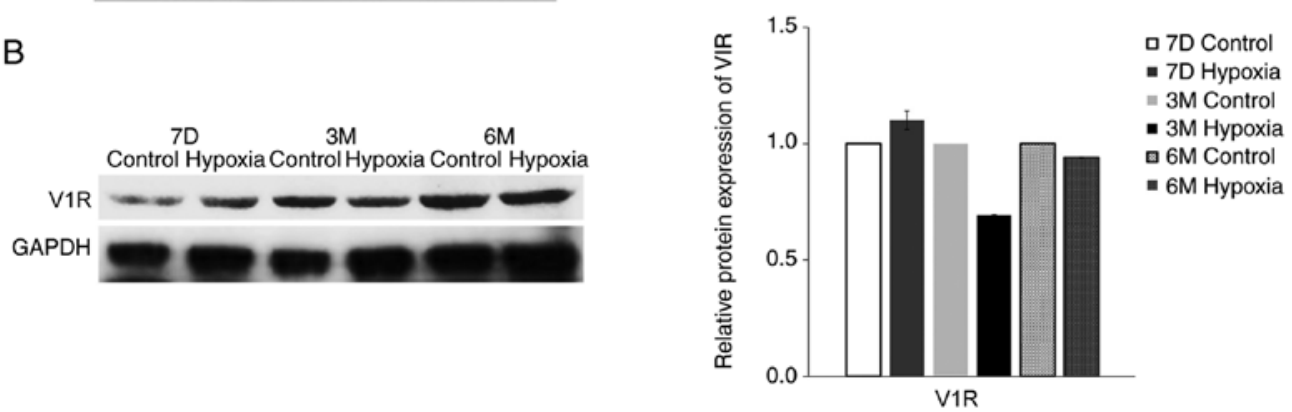

Figure 3. Maternal rats were exposed to continuous hypoxia from days 7-21 during pregnancy and the protein levels of (A) indicated proteins and the (B) target gene V1R in lung tissues of pups at 7D, 3M and 6M after birth were measured by western blot analysis. The expression of GAPDH was used for normalization. $\mathrm{n}=3$. Samples were each measured 3 times. Data are presented as mean \pm standard deviation. A two-way ANOVA and Bonferroni post hoc test were used to analyze the data between the control and hypoxia groups. ${ }^{*} \mathrm{P}<0.05$ and ${ }^{* *} \mathrm{P}<0.01$ vs. control. HIF-1 $\alpha$, hypoxia-inducible factor-1 $\alpha$; HIF-2 $\alpha$, hypoxia-inducible factor- $2 \alpha$; V1R, vasopressin type-1 receptor; V2R, vasopressin type-2 receptor; PIGF, placental growth factor; TGF- $\beta$, Transforming growth factor- $\beta$ 1; TNF- $\alpha$, tumor necrosis factor- $\alpha$; $\alpha$-SMA, $\alpha$-smooth muscle aorta; 7D, 7 days; 3M, 3 months; 6M, 6 months. 

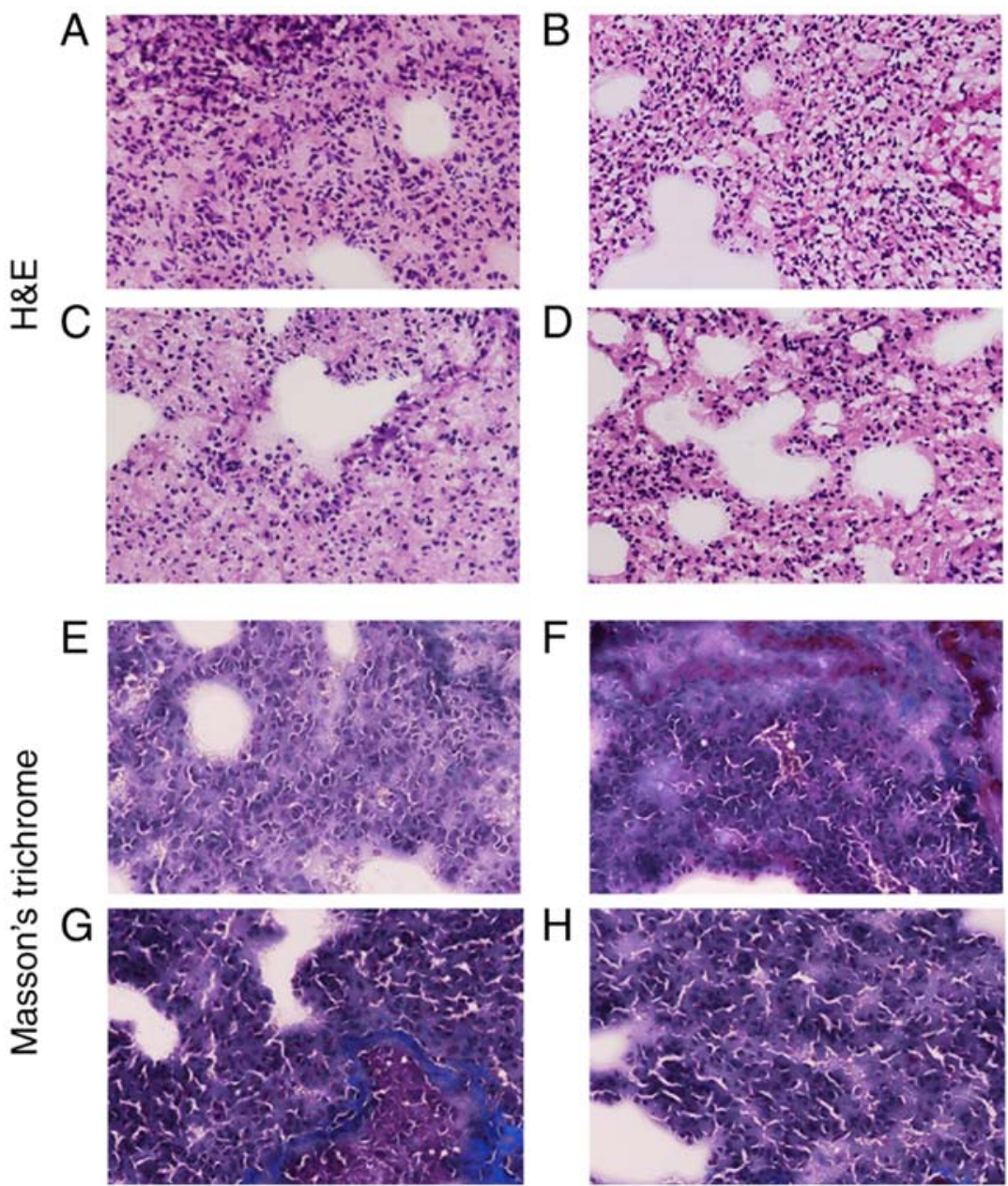

Figure 4. Hematoxylin \& eosin and Masson's trichrome staining of lung tissues (A) Control rats at 7 days. (B) Hypoxia rats at 7 days (C) Control rats at 3 months. (D) Hypoxia rats at 3 months. (E) Control rats at 7 days. (F) Hypoxia rats at 7 days. (G) Control rats at 3 months. (H) Hypoxia rats at 3 months. Magnification, $\mathrm{x} 400$

in the hypoxia group at 6 months after birth, V2R may be an indicator for PH in postnatal rats. However, the transcription levels of several other mRNAs were also significantly different between the hypoxia and control groups at 6 months after birth. In particular, HIF-1 $\alpha$, Ang-2, a-SMA mRNAs, and to a certain extent PIGF mRNAs, were expressed at significantly increased levels in pups from normoxic maternal rats compared with pups from hypoxic rats. Compared with their respective levels at birth, these mRNAs in the control group were all increased at 6 months. The protein expression levels, determined by western blotting, confirmed the mRNA expression results (Fig. 3). For example, the protein expression level of V2R in the lung tissue of the experimental group was 3.2-fold higher compared with the level in the control group, probably due to exposure to hypoxia in utero.

Blood vessels in the lungs of offspring rats are damaged following fetal hypoxia. H\&E (Fig. 4A-D) and Masson's (Fig. 4E-H) staining protocols of the tissue sections demonstrated that the lung tissues from hypoxia rats were significantly damaged due to the long-term hypoxia treatment, and the lung weight of newborns was significantly affected (Table III). $\alpha$-SMA immunohistochemical staining (Fig. 5) indicated that at 1 month after birth, the positive count of $\alpha$-SMA staining was increased in the lung tissue of rats in the hypoxia group compared with the controls. However, at 6 months, the positive count of $\alpha$-SMA staining decreased in the hypoxia rat group and inversely increased in the control group.

Changes in WT\% in offspring exposed to hypoxia. The WT\% was measured using sections stained with H\&E and Masson's stain (Fig. 4). Compared with the control group, the WT\% value of 7-day old pups in the hypoxia group was significantly increased compared with age-matched pups in the control group. However, as postnatal adjustment to normoxic conditions progressed, the vascular wall of the pups exposed to hypoxia became considerably thinner and the vascular diameter became larger (Fig. 6). The WT\% at 3 months after birth in the hypoxic animals was equivalent to that of the control pups. At 6 months, the value decreased further, to below the level in the control pups. By contrast, the WT\% values of the normoxia control pups showed no significant changes at 7 days, 3 months and 6 months after birth, demonstrating that the WT\% values of the pups exposed to hypoxia treatment during gestation were significantly smaller compared with those of the control pups at 6 months after birth. 
A

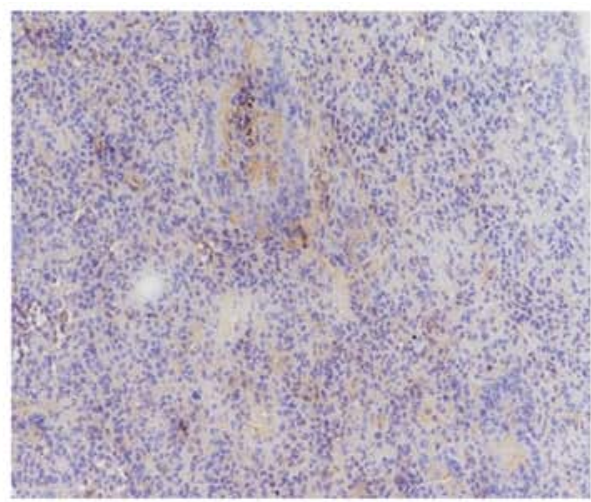

$\mathrm{C}$

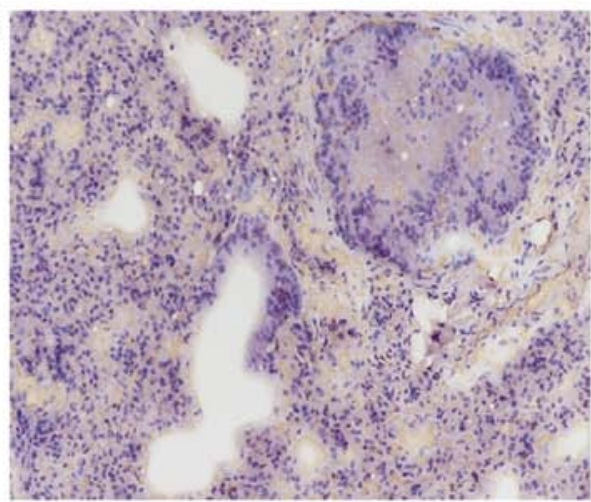

B

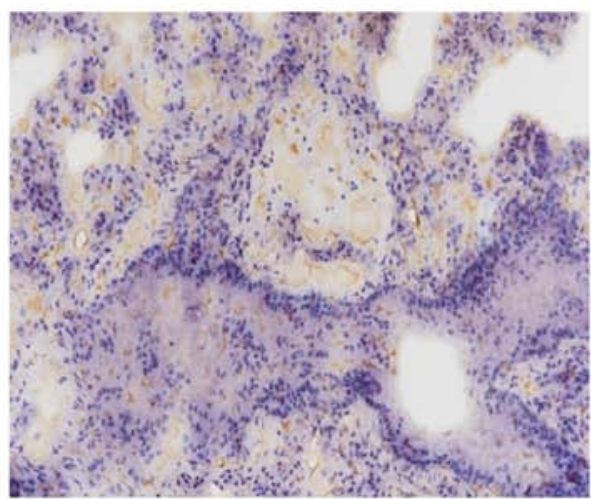

D

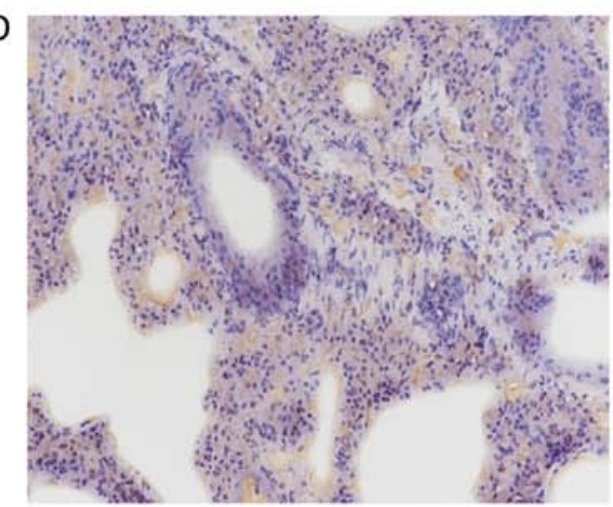

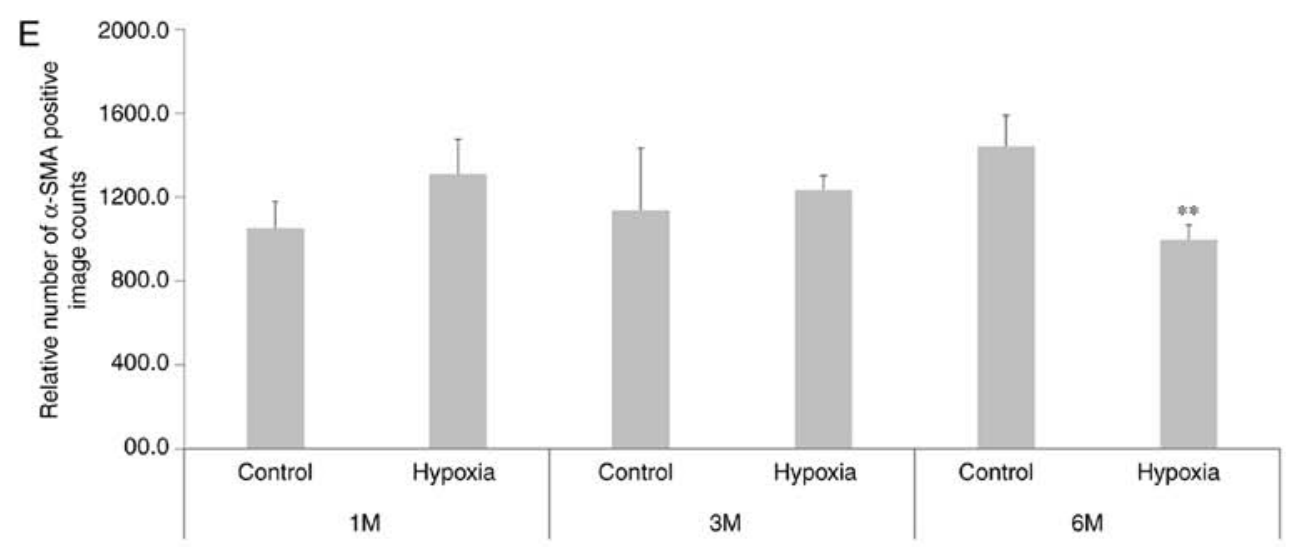

Figure 5. $\alpha$-SMA antibody staining. (A) Control rats at 1 month. (B) Hypoxic rats at $1 \mathrm{M}$, (C) Control rat at 3M. (D) Hypoxic rat at 3M. All images are at magnification x100. (E) Relative number of $\alpha$-SMA positive image counts at $1 M, 3 M$ and $6 M$. $n=3$. Samples were each measured 3 times. Data are presented as mean \pm standard deviation. A two-way ANOVA and Bonferroni post hoc test were used to analyze the data between the control and hypoxia groups. ${ }^{* *} \mathrm{P}<0.01$ vs. control. $\alpha$-SMA, $\alpha$-smooth muscle actin; $1 \mathrm{M}, 1$ month; $3 \mathrm{M}, 3$ months; $6 \mathrm{M}, 6$ months.

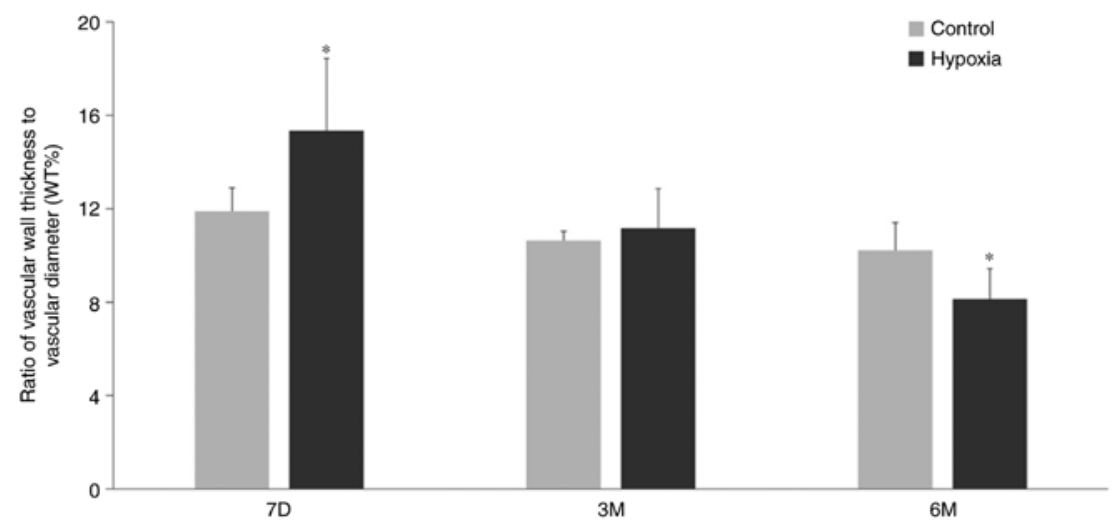

Figure 6. Vascular wall thickness and vascular diameter of offspring from control maternal rats and hypoxia-treated maternal rats during pregnancy. $\mathrm{n}=3$. Samples were each measured 3 times. Data are presented as mean \pm standard deviation. A two-way ANOVA and Bonferroni post hoc test were used to analyze the data between the control and hypoxia groups. ${ }^{*} \mathrm{P}<0.05$ vs. control. WT\%, ratio of vascular wall thickness to vascular diameter. 


\section{Discussion}

In the present study, persistent $\mathrm{PH}$ demonstrated to be caused in newborn rats by the exposure of the mother to a hypoxic environment for 3 weeks during pregnancy. $\mathrm{PH}$ is a complex disease reflected in physiological (vasoconstriction) or structural (arteriole remodeling and a decrease in pulmonary vessel density) changes. As the developmental stage of rats at birth is roughly equivalent to humans in utero at 3 months, the rat hypothalamus and pituitary gland at birth and up to 7 days after are still under development, which are critical central factors involved in blood pressure regulation of the animals. The present study identified that the expression of several angiogenesis-associated factors, including HIF-1 $\alpha$, HIF- $2 \alpha$, TGF- $\beta$, TNF- $\alpha$, PlGF, $\alpha$-SMA, Ang- 2 and V2R, were affected to different extents. Among these factors, which were all increased at birth, only V2R expression remained increased in pups exposed to hypoxia in utero until 6 months of age, compared with the control pups. The V2R expression levels in their lung tissue at both 3 months and 6 months was at least 2 -fold greater compared with those of normoxic control pups. These results suggest that intrauterine hypoxia may be responsible for the decreased expression levels of vascular factors in lung tissue and the decrease in the number of pulmonary vessels, as well as thinning of the pulmonary vascular walls, as observed in the tissue sections of 6-month-old offspring exposed to hypoxia in utero.

Activation of $\mathrm{V} 2 \mathrm{R}$ in cancer cells leads to a release of factors that decrease their metastatic potential $(33,34)$. Previous studies have demonstrated that long-term administration of the selective competitive V2R and kidney V1R receptor antagonist tolvaptan elicited positive effects on cardiac hemodynamics, and moderated LV remodeling and myocardial fibrosis in association with diuretic therapy $(35,36)$. Therefore, we hypothesized that continuous chronic V2R expression negatively affected the pulmonary vasculature in the experimental model of the present study.

HIF-1 $\alpha$ has been demonstrated to be a vital mediator in $\mathrm{PH}$ and to alleviate $\mathrm{PH}$ when knocked down by RNA interference in adult rats (10). In addition to HIF-1 $\alpha$, data concerning HIF- $2 \alpha$, a HIF-1 $\alpha$ homologue, has revealed that heterozygous deletion of HIF- $2 \alpha$ can attenuate $\mathrm{PH}$ induced by hypoxia in mice (23), which suggests that HIF-2 $\alpha$ also contributes to the development of hypoxic $\mathrm{PH}$. The present study identified that the levels of HIF- $1 \alpha$ and $2 \alpha$ were raised in hypoxia pups at birth; however, they were decreased compared with control levels after 3 months. At 6 months, both HIF-1 $\alpha$ and HIF- $2 \alpha$ levels were significantly decreased, suggesting that it is unlikely that activation of HIF- $1 \alpha$ and $2 \alpha$ is the primary cause of $\mathrm{PH}$. It was also observed that V2R, which is closely related to HF, was highly expressed in lung tissues of hypoxia rats following birth. Therefore, we hypothesized that V2R is not only an indicator of the potential risk of $\mathrm{PH}$ in infants suffering from hypoxia, but may actually be directly involved in $\mathrm{PH}$ and could be considered a target for treatment. Goto et al (21) reported that tolvaptan may contribute to RV remodeling in a hypoxic rat model and had a detrimental effect on pulmonary heart hypertension. However, their model used the vascular endothelial growth factor receptor inhibitor SU5416 in combination with hypoxia to induce $\mathrm{PH}$ on 6-week-old male
Sprague-Dawley rats, and then evaluated the effects, which is unsuitable for a comparison with the results of the present study, which analyzed the effects of hypoxic conditions during pregnancy on the offspring of rats without additional treatments. Conversely, a few previous studies observed that tolvaptan was efficient in treating patients with right heart failure due to $\mathrm{PH}(37,38)$.

A limitation of the present study is that hypoxia related parameters, such as blood vessel growth pattern, and pulmonary hemodynamic changes were not measured in the pregnant rats after hypoxia, which might have provided additional valuable information for the prediction of $\mathrm{PH}$ in their offspring.

In conclusion, the present study identified that the V2R expression levels in newborn rats induced by maternal exposure to hypoxia were sustained to significantly increased levels compared with the pups not exposed to maternal hypoxia. V2R may be a useful marker for the diagnosis and prognosis of $\mathrm{PH}$, and for the risk of right heart failure in fetuses suffering from hypoxia during pregnancy.

\section{Acknowledgements}

Not applicable.

\section{Funding}

Funding for the present study was provided by grants from the National Natural Science Foundation of China (grant nos. 81370181 and 81770089) and Zhenjiang Key Research and Development Program of China (grant no. SH2018077).

\section{Availability of data and materials}

All data generated or analyzed during this study are included in this published article.

\section{Authors' contributions}

$\mathrm{KH}$ was responsible for the conception and design of the study. HD, YL, PL, HH, LZ, JY and YX were responsible for acquisition and analysis of the data. MX, HH and $\mathrm{LZ}$ were responsible for the statistical analysis. HD, KH, HH, PL and MX drafted the manuscript; YL, LZ, JY and YX revised the manuscript critically. All authors read and approved the final manuscript.

\section{Ethics approval and consent to participate}

All experiments on rats were conducted according to the guidelines for the Humane Treatment of Laboratory Animals (Ministry of Science and Technology of the People's Republic of China, Policy No. 2006 398) and further approval for this study was waived by the ethics committee of the Affiliated People's Hospital of Jiangsu University.

\section{Patient consent for publication}

Not applicable. 


\section{Competing interests}

The authors declare that they have no competing interests.

\section{References}

1. Mathew B and Lakshminrusimha S: Persistent pulmonary hypertension in the newborn. Children (Basel) 4: 63, 2017.

2. Rudolph AM and Yuan S: Response of the pulmonary vasculature to hypoxia and $\mathrm{H}^{+}$ion concentration changes. J Clin Invest 45 399-411, 1966.

3. Tabima DM, Frizzell S and Gladwin MT: Reactive oxygen and nitrogen species in pulmonary hypertension. Free Radic Biol Med 52: 1970-1986, 2012.

4. Galiè N, Hoeper MM, Humbert M, Torbicki A, Vachiery JL, Barbera JA, Beghetti M, Corris P, Gaine S, Gibbs JS, et al: Guidelines for the diagnosis and treatment of pulmonary hypertension: The task force for the diagnosis and treatment of pulmonary hypertension of the European society of cardiology (ESC) and the European respiratory society (ERS), endorsed by the international society of heart and lung transplantation (ISHLT). Eur Heart J 30: 2493-2537, 2009.

5. Simonneau G, Gatzoulis MA, Adatia I, Celermajer D, Denton C, Ghofrani A, Gomez Sanchez MA, Krishna Kumar R, Landzberg M, Machado RF, et al: Updated clinical classification of pulmonary hypertension. J Am Coll Cardiol 62: D34-D41, 2013.

6. Rosenkranz S: Pulmonary hypertension 2015: Current definitions, terminology, and novel treatment options. Clin Res Cardiol 104: 197-207, 2015

7. Delaney $\mathrm{C}$ and Cornfield DN: Risk factors for persistent pulmonary hypertension of the newborn. Pulm Circ 2: 15-20, 2012.

8. Storme L, Aubry E, Rakza T, Houeijeh A, Debarge V, Tourneux P, Deruelle P, Pennaforte T and French Congenital Diaphragmatic Hernia Study G: Pathophysiology of persistent pulmonary hypertension of the newborn: Impact of the perinatal environment. Arch Cardiovasc Dis 106: 169-177, 2013.

9. Xu XF, Ma XL, Shen Z, Wu XL, Cheng F and Du LZ: Epigenetic regulation of the endothelial nitric oxide synthase gene in persistent pulmonary hypertension of the newborn rat J Hypertens 28: 2227-2235, 2010.

10. Li Y, Shi B, Huang L, Wang X, Yu X, Guo B and Ren W: Suppression of the expression of hypoxia-inducible factor-1alpha by RNA interference alleviates hypoxia-induced pulmonary hypertension in adult rats. Int J Mol Med 38: 1786-1794, 2016.

11. Ambalavanan N, Nicola T, Hagood J, Bulger A, Serra R, Murphy-Ullrich J, Oparil S and Chen YF: Transforming growth factor-beta signaling mediates hypoxia-induced pulmonary arterial remodeling and inhibition of alveolar development in newborn mouse lung. Am J Physiol Lung Cell Mol Physiol 295: L86-L95, 2008.

12. Özpelit E, Akdeniz B, Özpelit ME, Tas S, Bozkurt S, Tertemiz KC, Sevinc C and Badak Ö: Prognostic value of neutrophil-to-lymphocyte ratio in pulmonary arterial hypertension. J Int Med Res 43: 661-671, 2015.

13. Seyfarth HJ, Sack U, Gessner C and Wirtz H: Angiogenin, bFGF and VEGF: angiogenic markers in breath condensate of patients with pulmonary hypertension. Pneumologie 69: 207-211, 2015 (In German).

14. Rameh V and Kossaify A: Role of biomarkers in the diagnosis, risk assessment, and management of pulmonary hypertension. Biomark Insights 11: 85-89, 2016.

15. Muttukrishna S, Swer M, Suri S, Jamil A, Calleja-Agius J, Gangooly S, Ludlow H, Jurkovic D and Jauniaux E: Soluble flt-1 and PIGF: New markers of early pregnancy loss? PLoS One 6: e18041, 2011.

16. Cohen SS, Powers BR, Lerch-Gaggl A, Teng RJ and Konduri GG: Impaired cerebral angiogenesis in the fetal lamb model of persistent pulmonary hypertension. Int J Dev Neurosci 38 : 113-118, 2014.

17. Xu XF, Gu WZ, Wu XL, Li RY and Du LZ: Fetal pulmonary vascular remodeling in a rat model induced by hypoxia and indomethacin. J Matern Fetal Neonatal Med 24: 172-182, 2011.
18. Haworth SG and Reid L: Persistent fetal circulation: Newly recognized structural features. J Pediatr 88: 614-620, 1976.

19. Haworth SG: Pulmonary vascular remodeling in neonatal pulmonary hypertension. State of the art. Chest 93: 133S-138S, 1988.

20. Pluchart H, Khouri C, Blaise S, Roustit M and Cracowski JL: Targeting the prostacyclin pathway: Beyond pulmonary arterial hypertension. Trends Pharmacol Sci 38: 512-523, 2017.

21. Goto I, Dohi K, Ogihara Y, Okamoto R, Yamada N, Mitani Y and Ito M: Detrimental impact of vasopressin V2 receptor antagonism in a su5416/hypoxia/normoxia-exposed rat model of pulmonary arterial hypertension. Circul J 80: 989-997, 2016.

22. Ikeda $T$, Iwanaga $Y$, Watanabe $H$, Morooka $H$, Akahoshi $Y$, Fujiki $\mathrm{H}$ and Miyazaki S: Effects of long-term blockade of vasopressin receptor types 1a and 2 on cardiac and renal damage in a rat model of hypertensive heart failure. J Cardiovasc Pharmacol 66: 487-496, 2015.

23. Stayer SA and Liu Y: Pulmonary hypertension of the newborn. Best Pract Res Clin Anaesthesiol 24: 375-386, 2010.

24. Teng RJ and Wu TJ: Persistent pulmonary hypertension of the newborn. J Formos Med Assoc 112: 177-184, 2013.

25. Roofthooft MT, Elema A, Bergman KA and Berger RM: Patient characteristics in persistent pulmonary hypertension of the newborn. Pulm Med 2011: 858154, 2011.

26. Niermeyer S: Cardiopulmonary transition in the high altitude infant. High Alt Med Biol 4: 225-239, 2003.

27. Niermeyer S: Going to high altitude with a newborn infant. High Alt Med Biol 8: 117-123, 2007.

28. Guidance on the Care of Laboratory Animals: (2006) No. 398 , Ministry of Science and Technology, 2006.

29. Wang Z, Huang Z, Lu G, Lin L and Ferrari M: Hypoxia during pregnancy in rats leads to early morphological changes of atherosclerosis in adult offspring. Am J Physiol Heart Circ Physiol 296: H1321-H1328, 2009.

30. Canadian Council on Animal Care. CCAC guidelines on: Euthanasia of animals used in science, 2010. http://www.ccac. ca/Documents/Standards/Guidelines/Euthanasia.pdf. Accessed February 21, 2016.

31. Albert Einstein College of Medicine Institute for Animal Studies. Recommended Methods of Anesthesia, Analgesia, and Euthanasia for Laboratory Animal Species, 2014.

32. Livak KJ and Schmittgen TD: Analysis of relative gene expression data using real-time quantitative PCR and the 2(-Delta Delta C(T)) method. Methods 25: 402-408, 2001

33. Pifano M, Garona J, Capobianco CS, Gonzalez N, Alonso DF and Ripoll GV: Peptide agonists of vasopressin V2 receptor reduce expression of neuroendocrine markers and tumor growth in human lung and prostate tumor cells. Front Oncol 7: 11, 2017.

34. Ripoll GV, Garona J, Hermo GA, Gomez DE and Alonso DF Effects of the synthetic vasopressin analog desmopressin in a mouse model of colon cancer. Anticancer Res 30: 5049-5054, 2010.

35. Morooka H, Iwanaga Y, Tamaki Y, Takase T, Akahoshi Y, Nakano Y, Fujiki $\mathrm{H}$ and Miyazaki S: Chronic administration of oral vasopressin type 2 receptor antagonist tolvaptan exerts both myocardial and renal protective effects in rats with hypertensive heart failure. Circ Heart Fail 5: 484-492, 2012.

36. Yamazaki T, Izumi Y, Nakamura Y, Yamashita N, Fujiki H, Osada-Oka M, Shiota M, Hanatani A, Shimada K, Iwao H and Yoshiyama M: Tolvaptan improves left ventricular dysfunction after myocardial infarction in rats. Circ Heart Fail 5: 794-802, 2012.

37. Tamura Y, Kimura M, Takei M, Ono T, Kuwana M, Satoh T, Fukuda K and Humbert M: Oral vasopressin receptor antagonist tolvaptan in right heart failure due to pulmonary hypertension. Eur Respir J 46: 283-286, 2015.

38. Joko Y, Ikemura N, Miyata K, Shiraishi Y, Tanaka H, Yoshida T, Ikegami Y, Fuse J, Sakamoto M and Momiyama Y: Efficacy of tolvaptan in a patient with right-sided heart failure and renal dysfunction refractory to diuretic therapy. J Cardiol Cases 9: 226-229, 2014

This work is licensed under a Creative Commons Attribution-NonCommercial-NoDerivatives 4.0 International (CC BY-NC-ND 4.0) License. 